\title{
Screening for Subtelomeric Rearrangements in Thai Patients with Intellectual Disabilities Using FISH and Review of Literature on Subtelomeric FISH in 15,591 Cases with Intellectual Disabilities
}

\author{
Chariyawan Charalsawadi, ${ }^{1}$ Jariya Khayman, ${ }^{1}$ \\ Verayuth Praphanphoj, ${ }^{2}$ and Pornprot Limprasert ${ }^{1}$ \\ ${ }^{1}$ Department of Pathology, Faculty of Medicine, Prince of Songkla University, Songkhla 90110, Thailand \\ ${ }^{2}$ Medical Genetics Center, Bangkok 10220, Thailand \\ Correspondence should be addressed to Pornprot Limprasert; lpornpro@yahoo.com
}

Received 11 May 2016; Revised 8 August 2016; Accepted 9 August 2016

Academic Editor: Norman A. Doggett

Copyright (C) 2016 Chariyawan Charalsawadi et al. This is an open access article distributed under the Creative Commons Attribution License, which permits unrestricted use, distribution, and reproduction in any medium, provided the original work is properly cited.

\begin{abstract}
We utilized fluorescence in situ hybridization (FISH) to screen for subtelomeric rearrangements in 82 Thai patients with unexplained intellectual disability (ID) and detected subtelomeric rearrangements in 5 patients. Here, we reported on a patient with $\operatorname{der}(20) t(X ; 20)(\mathrm{p} 22.3 ; \mathrm{q} 13.3)$ and a patient with $\operatorname{der}(3) \mathrm{t}(\mathrm{X} ; 3)(\mathrm{p} 22.3 ; \mathrm{p} 26.3)$. These rearrangements have never been described elsewhere. We also reported on a patient with $\operatorname{der}(10) t(7 ; 10)(\mathrm{p} 22.3 ; \mathrm{q} 26.3)$, of which the same rearrangement had been reported in one literature. Well-recognized syndromes were detected in two separated patients, including $4 \mathrm{p}$ deletion syndrome and $1 \mathrm{p} 36$ deletion syndrome. All patients with subtelomeric rearrangements had both ID and multiple congenital anomalies (MCA) and/or dysmorphic features (DF), except the one with der(20)t(X;20), who had ID alone. By using FISH, the detection rate of subtelomeric rearrangements in patients with both ID and MCA/DF was $8.5 \%$, compared to $2.9 \%$ of patients with only ID. Literature review found 28 studies on the detection of subtelomeric rearrangements by FISH in patients with ID. Combining data from these studies and our study, 15,591 patients were examined and 473 patients with subtelomeric rearrangements were determined. The frequency of subtelomeric rearrangements detected by FISH in patients with ID was 3\%. Terminal deletions were found in $47.7 \%$, while unbalanced derivative chromosomes were found in $47.9 \%$ of the rearrangements.
\end{abstract}

\section{Introduction}

An intellectual disability (ID) is a condition wherein the development of the mentality is arrested or incomplete, which contributes to the impairment of the overall level of intelligence, including cognitive, language, motor, and social abilities [1]. ID affects $1 \%$ to $3 \%$ of the global population. Besides environmental factors, genetic factors are a significant cause of ID. More than half of all patients with ID are categorized as having an unexplained ID, with subtelomeric rearrangements having been observed in a number of these patients, ranging between 0 and $29.4 \%$ [2].
The subtelomere is a region between chromosomespecific sequences and telomeric caps of each chromosome. This region is located in close proximity to a gene-rich area. Due to sequence homology between subtelomeres of different chromosomes, it can facilitate recombination and may subsequently result in detrimental effects, that is, promoting disease-causing chromosomal rearrangements [3].

Subtelomeric rearrangements can be detected by various methods. In this study, we utilized fluorescent in situ hybridization (FISH) method with probes specific to the subtelomere region of all chromosome ends to screen for subtelomeric rearrangements in patients with unexplained 
ID. We identified new rearrangements and described clinical entities of patients with the rearrangements.

\section{Subjects and Methods}

Inclusion criteria for subject recruitment were patients with ID of unknown causes, with or without multiple congenital anomalies (MCA) and/or dysmorphic features (DF) and with normal G-banding chromosome analysis result at the 450550 bands' levels. All patients who met these inclusion criteria were referred from clinicians at Rajanukul Institute. The institute is the governmental agency under the Department of Mental Health, Ministry of Public Health, providing medical care primarily for individuals with intellectual and developmental disabilities. Clinical features of some patients may be limited as we collected data from the laboratory order form. A total of 82 Thai patients with ID were recruited to the study. These patients included 50 males and 32 females aged between 1 year and 39 years (mean age being 4 years). ID without MCA/DF was present in 35 cases ( 22 males and 13 females), and ID with MCA/DF was present in 47 cases (28 males and 19 females). This study was a one-year prospective study that was conducted between the years of 2005 and 2006.

We performed subtelomeric FISH analysis on metaphase spreads obtained from lymphocyte cultures, which were initiated and harvested following a standard protocol. The FISH probes used in this study were constructed using bacterial artificial chromosome (BAC) as well as P1-derived artificial chromosome (PAC) clones. These clones contained 41 different subtelomeric-specific sequences for all human chromosome ends, located within $2 \mathrm{Mb}$ distance from the telomere. For the FISH analysis of each patient, the p-arm and q-arm probes of each chromosome were denatured and hybridized onto the denatured metaphase spreads. FISH was performed following a standard protocol. We examined at least 10 informative metaphase spreads for each chromosome. For patients with detected subtelomeric rearrangements, G-banding karyotype and FISH analyses using the same probes were carried out in parental blood when available. In addition, because the subtelomeric probe for the short arms of both $\mathrm{X}$ and $\mathrm{Y}$ chromosomes is specific to the pseudoautosomal region, the other $\mathrm{X}$ - and Y-specific probes were used to distinguish the chromosomes. This study was approved by the ethical committee of the Ministry of Public Health.

\section{Results}

We detected 5 subtelomeric rearrangements in 5 patients. The frequency of subtelomeric rearrangements in our study was $6.1 \%$. Two patients had deletions, including one with $\operatorname{del}(4)(\mathrm{p} 16.3)$ (Patient 1, Figure 1(a)) and one with $\operatorname{del}(1)(\mathrm{p} 36.3) \mathrm{dn}$ (Patient 2, Figure 1(b)). The other three patients had derivative chromosomes, including one with $\operatorname{der}(10) t(7 ; 10)(\mathrm{p} 22.3 ; \mathrm{q} 26.3)$ (Patient 3, Figure 1(c)), one with $\operatorname{der}(20) t(X ; 20)(p 22.3 ; q 13.3)$ (Patient 4, Figure 1(d)), and one with $\operatorname{der}(3) t(X ; 3)(p 22.3 ; p 26.3) d n$ (Patient 5, Figure 1(e)). Two patients had moderate degrees of ID, and 3 patients had severe degrees of ID, and those 3 patients included 2 patients with deletions. MCA/DF were observed in almost all of the patients with subtelomeric rearrangements, except a patient with $\operatorname{der}(20) t(X ; 20)$, who had only minor DF (Table 1$)$. The frequency of subtelomeric rearrangements in patients with ID and MCA/DF was $8.5 \%$, while in patients with only ID it was $2.9 \%$.

\section{Discussion}

4.1. Detection of Subtelomeric Rearrangements. Subtelomeric rearrangements can be detected using various methods, such as FISH $[4,26]$, multiplex ligation-dependent probe amplification (MLPA) [32-34], and a microarray-based method $[35,36]$. The latter method allows the whole human genome to be scanned at high resolution in a single experiment. As a result, not only cryptic subtelomeric rearrangements but also other unbalanced chromosomal abnormalities can be detected, such as interstitial deletions, duplications, and unbalanced translocations. However, FISH has the advantage of providing an instant location of certain rearrangements, such as insertions, inversions, and balanced translocations, which cannot be achieved by the microarray-based method.

We reviewed literature on subtelomeric rearrangements in individuals with ID, which were detected by subtelomeric FISH (Table 2). We search for articles in PubMed using the following keywords: subtelomeric FISH, intellectual disability, developmental delay, and mental retardation. We excluded studies from authors that did not provide details regarding number of the patients with ID, studies when FISH was performed to confirm findings of other molecular methods, studies where a selected panel of subtelomeric probes was used in each case, and extended studies with additional cases from the previous populations. We found 28 publications on the detection of subtelomeric rearrangements by FISH in patients with ID. Combining data from these studies (15,509 patients) and our study (82 patients), 15,591 patients with ID were examined by FISH and 474 subtelomeric rearrangements were identified in 473 patients. There was one patient with 2 rearrangements. Frequencies of subtelomeric rearrangements ranged from 0 to $20 \%$, with an average of $3 \%$ $(473 / 15,591)$ (Table 2). Familial variants and possible variants were found in approximately $1.0 \%(149 / 15,591)$ (Table 3$)$. One of the most common variants is the $\operatorname{del}(2)$ (qter), in which the detection of a deletion depends on the subtelomeric probe used. In this literature review, we found that frequency of the $\operatorname{del}(2)$ (qter) was $42 \%$ of all variants (63/149). It is important to do parental analysis with the same subtelomeric probe when an abnormality is detected in a patient to determine the clinical significance of the finding.

We divided subtelomeric rearrangements into 3 categories: (1) deletion, (2) derivative, and (3) others. The latter category included duplications, insertions, inversions, isochromosome, and balanced translocations. Deletions were found in approximately $47.7 \%$ (226/474), while derivative chromosomes were found in approximately 47.9\% (227/474) (Figure 2). The most frequent deletions ( $>5 \%$ of all deletions) involved chromosomes 1p, 22q, 9q, and 4p (Figure 3(a)). The derivative category was composed of an unbalanced translocation with both deletion and duplication of subtelomere regions, an unbalanced translocation with duplication of 


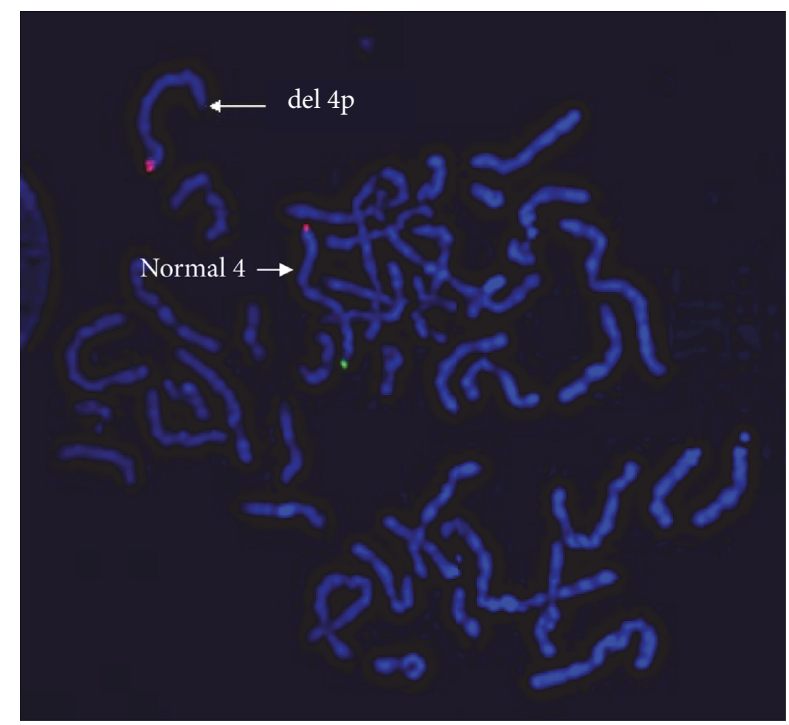

Green: 4p

Red: $4 \mathrm{q}$

(a) Patient 1

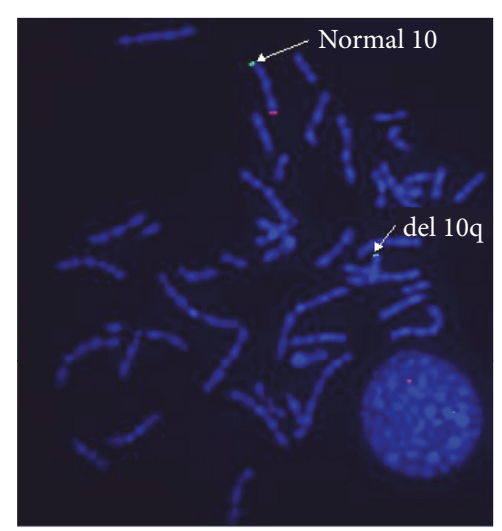

Green: 10p

Red: 10q

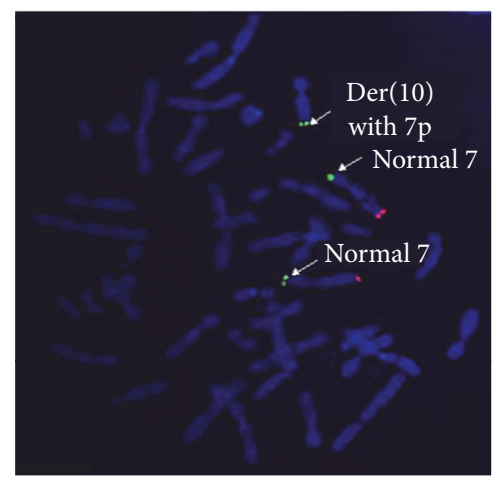

Green: $7 \mathrm{p}$ Red: 7q

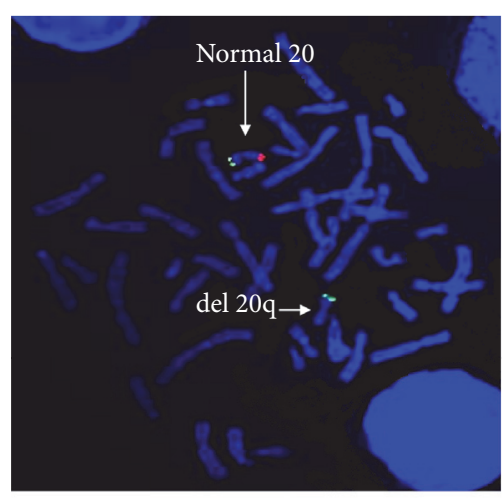

Green: 20p

Red: $20 q$

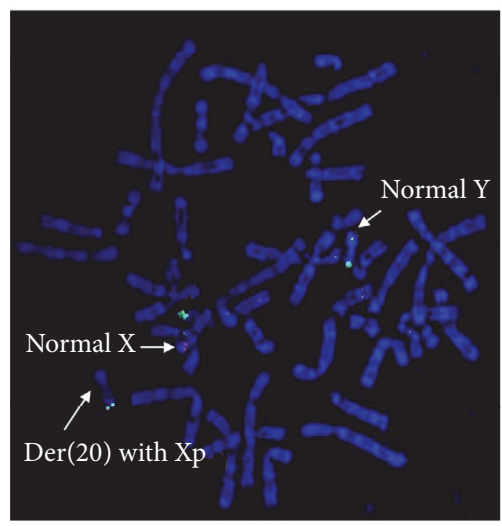

Green: Xp, Yp

Red: Xq

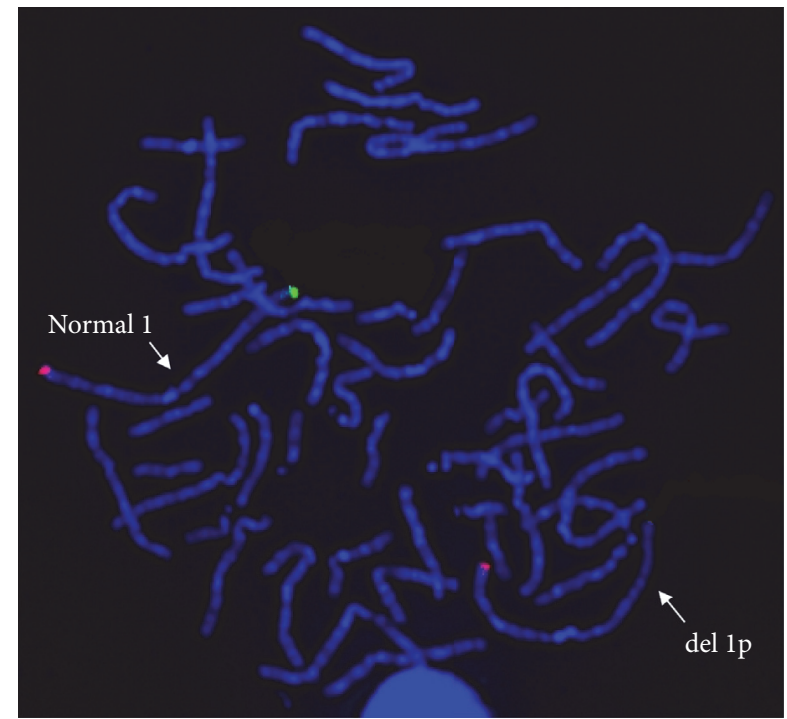

Green: 1p

Red: 1q

(b) Patient 2

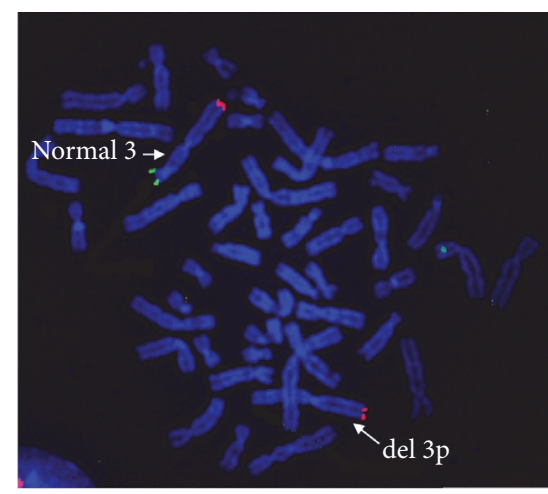

Green: $3 p$

Red: $3 q$

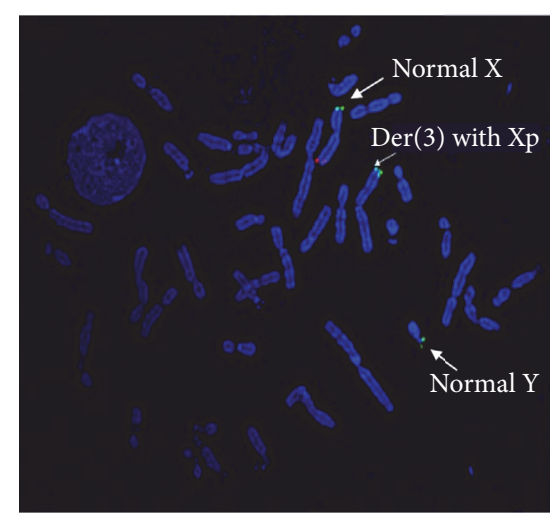

Green: Xp, Yp

Red: $\mathrm{Xq}$

(c) Patient 3

(d) Patient 4

(e) Patient 5

FIGURE 1: FISH results of 5 patients with subtelomeric rearrangements. 


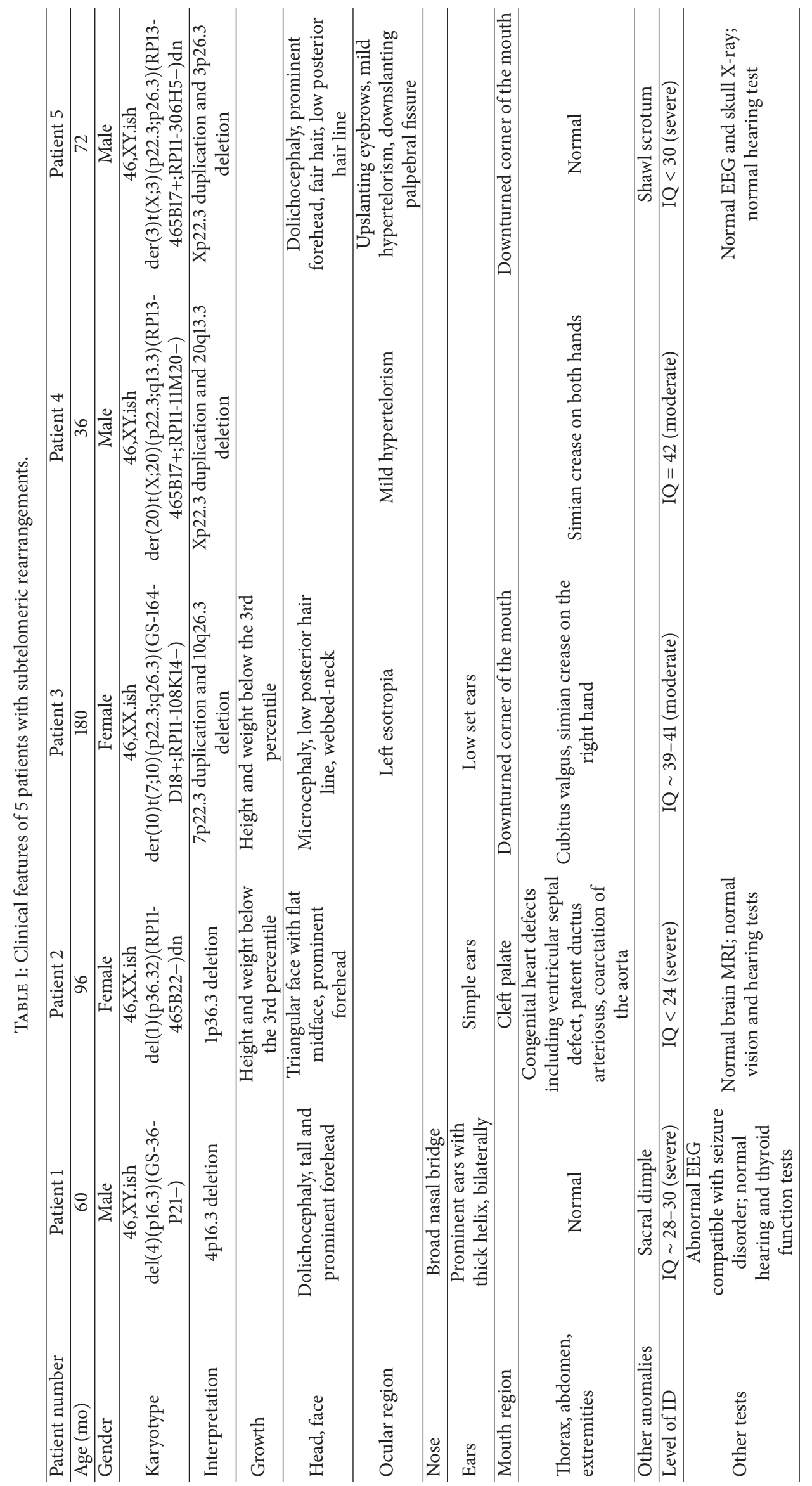




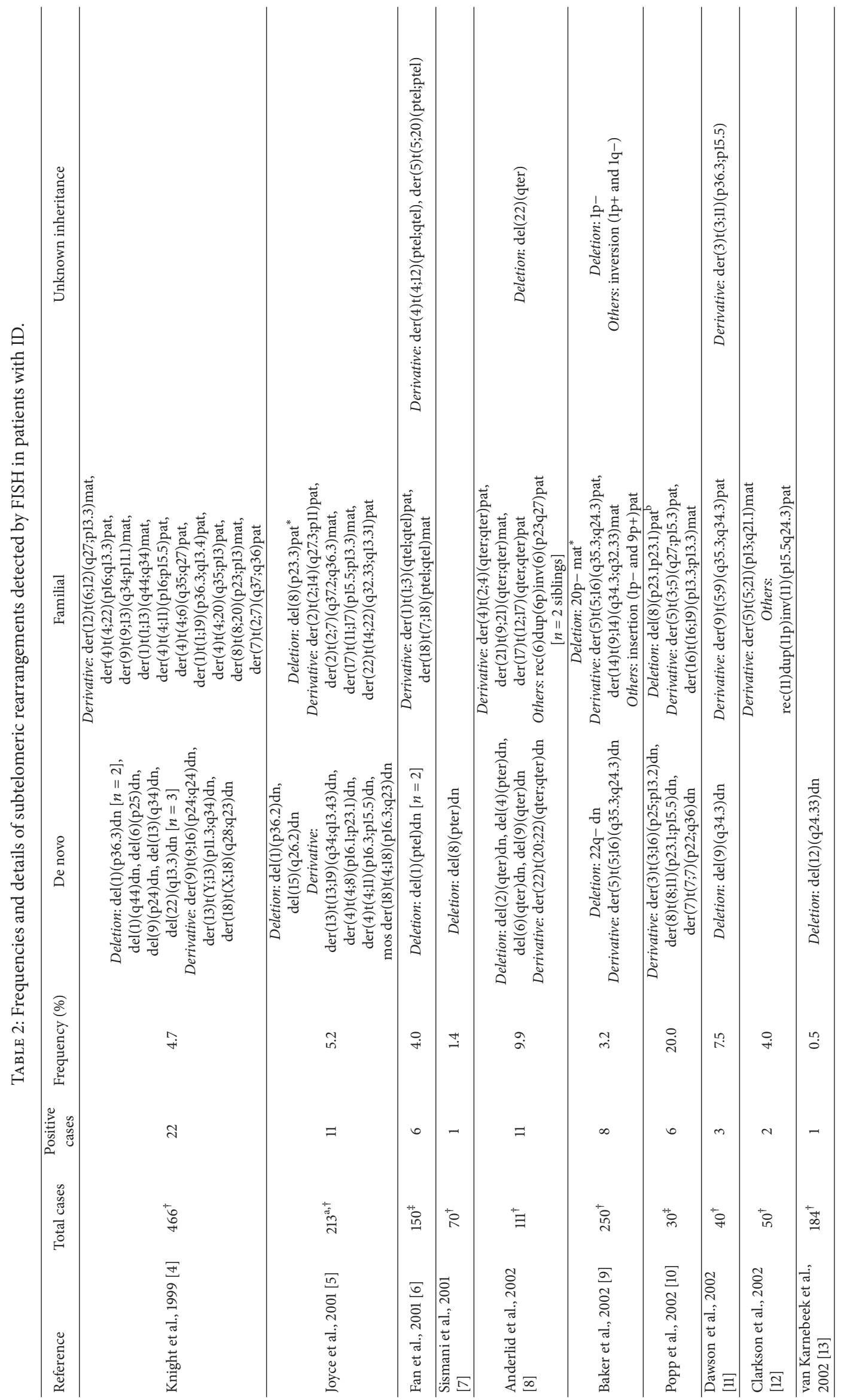




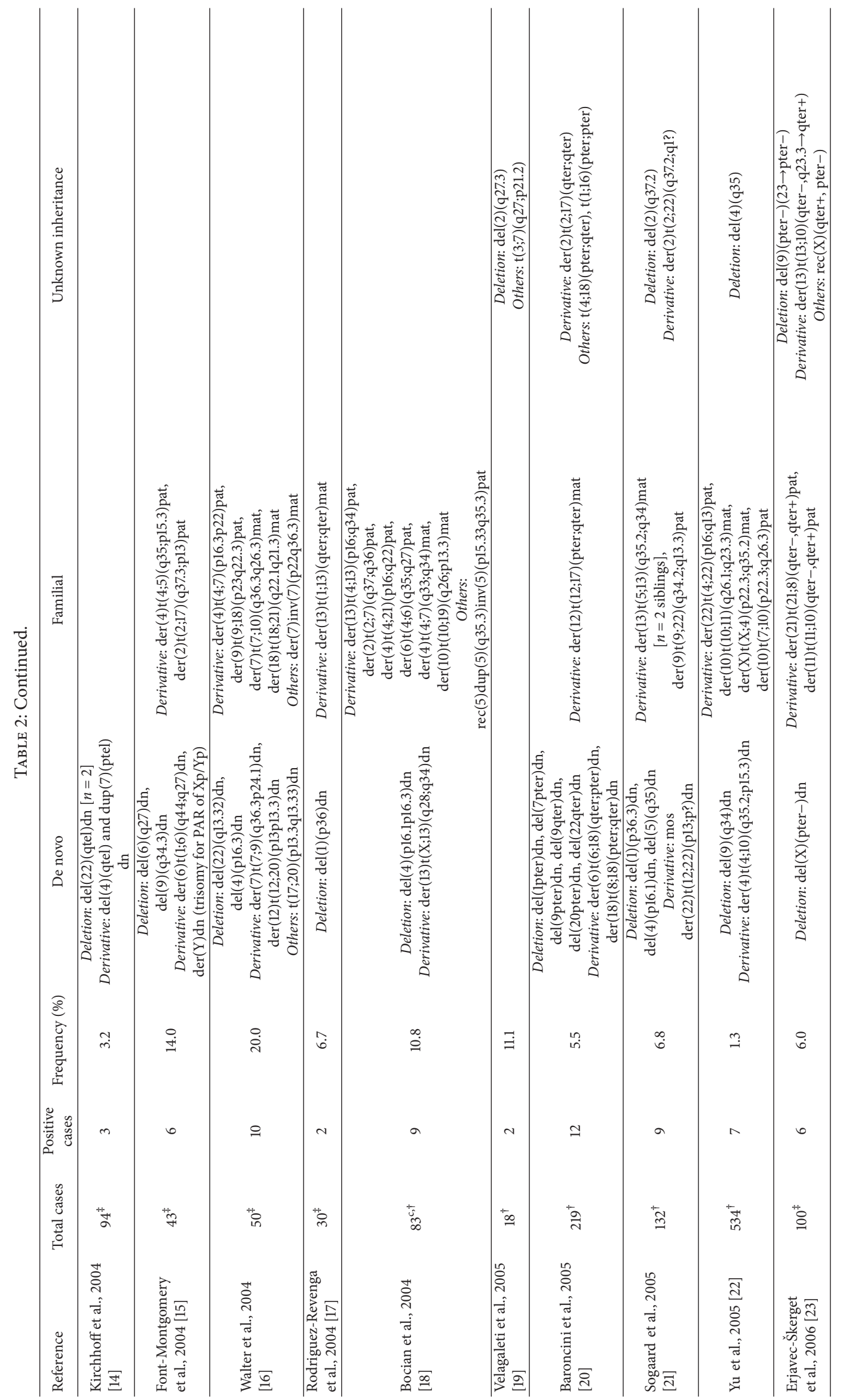




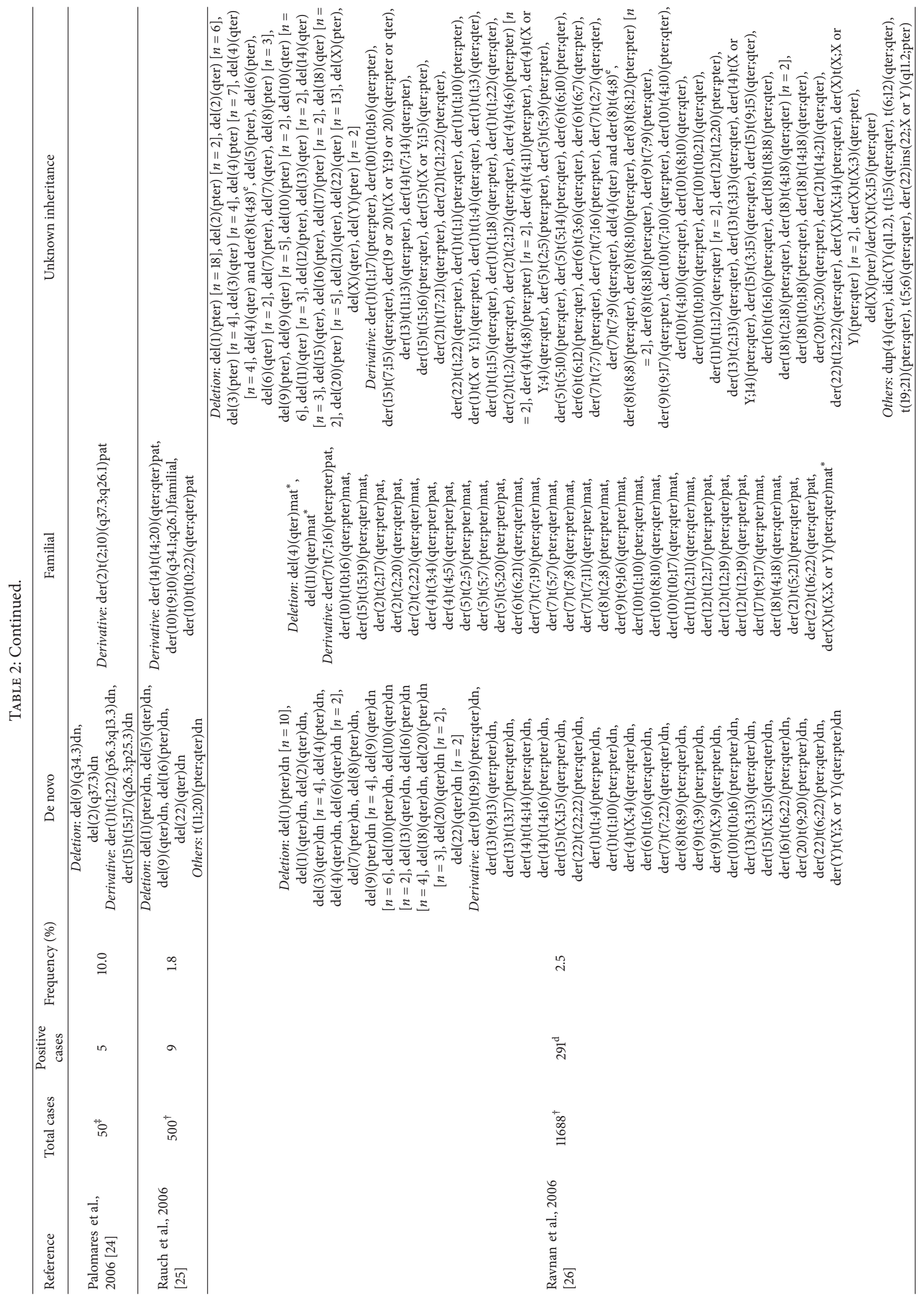




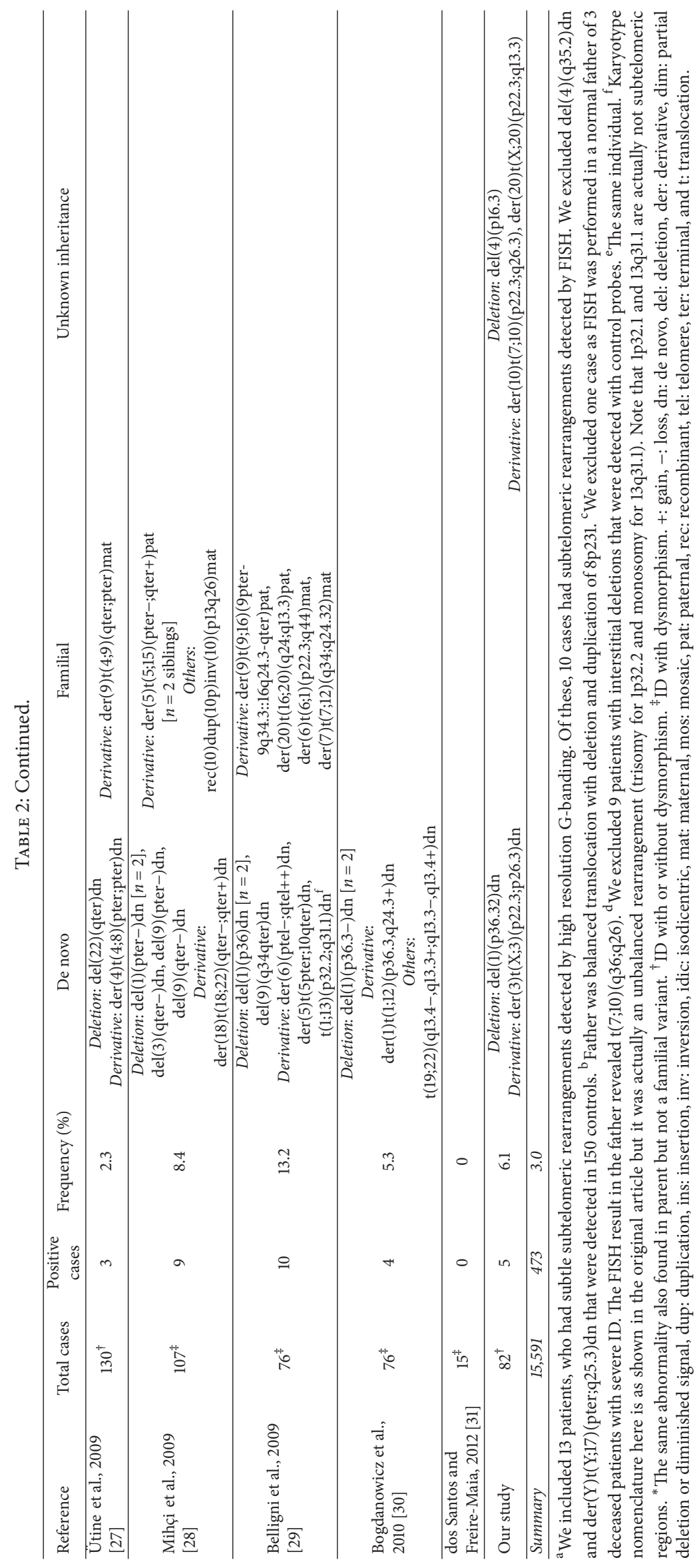




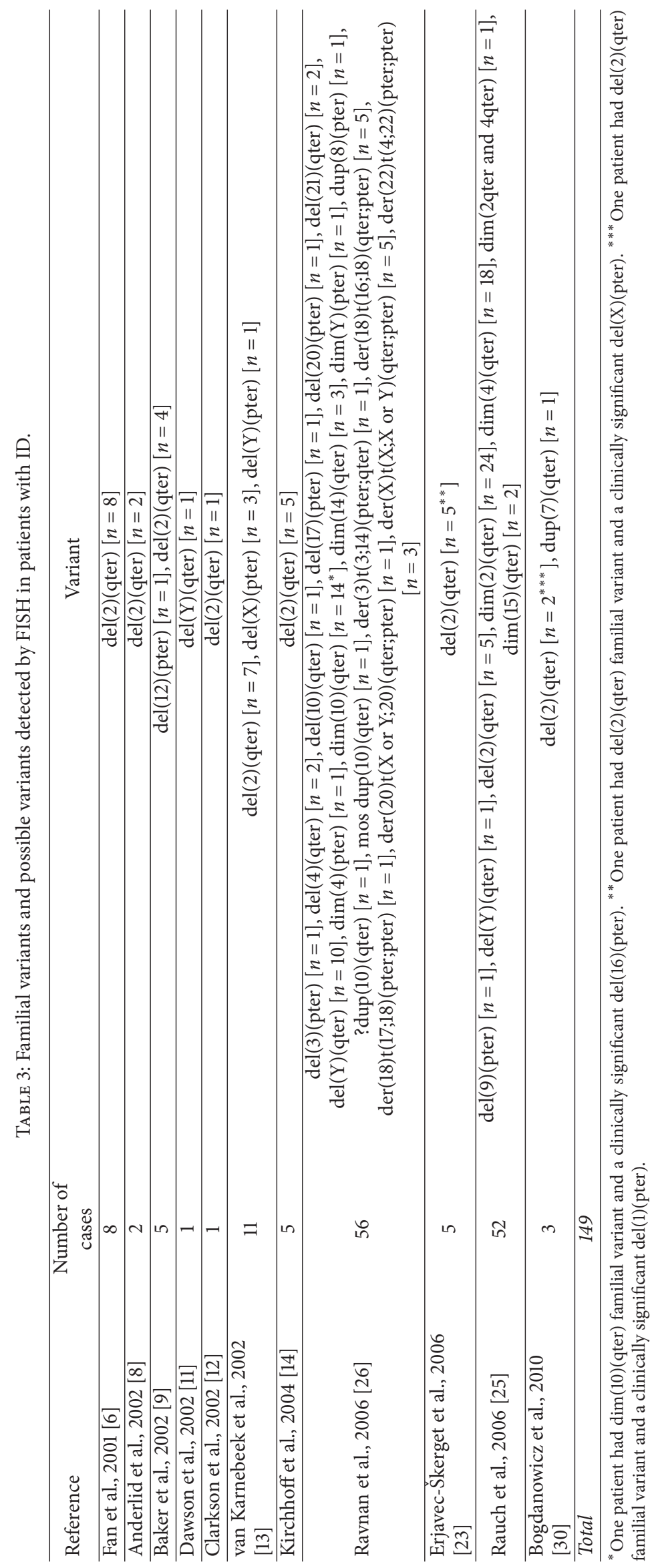




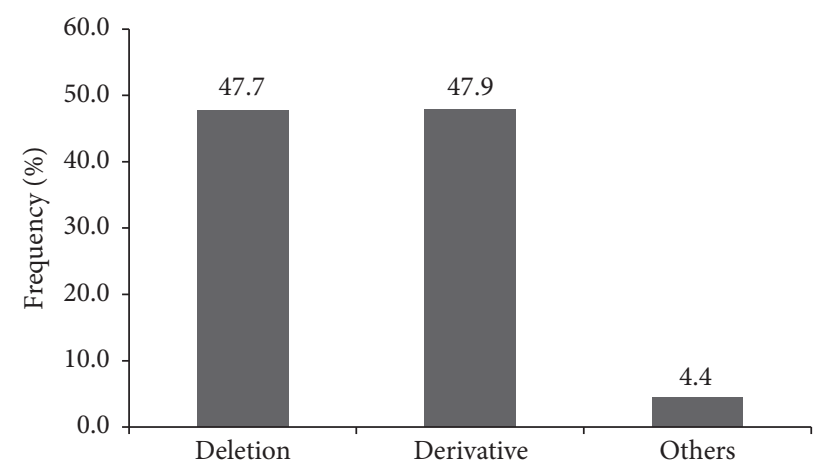

FIGURE 2: Frequency (\%) of each category of subtelomeric rearrangements.

a subtelomere region onto the short arm of acrocentric chromosome, and an unbalanced translocation with only duplication or deletion of a subtelomere region detected. Monosomies associated with unbalanced derivative chromosomes frequently involved chromosomes $4 \mathrm{p}, 10 \mathrm{q}, 2 \mathrm{q}, 5 \mathrm{p}, 13 \mathrm{q}, 18 \mathrm{q}$, and $7 \mathrm{q}$ (frequency $>5 \%$ of all the monosomies) (Figure $3(\mathrm{~b})$ ). In this study, we detected subtelomeric rearrangements in $6.1 \%$ of the patients. Deletions of chromosomes $1 \mathrm{p}$ and $4 \mathrm{p}$, which were among the most frequently detected deletions, were also detected in our study.

Attempts have been made to increase the diagnostic yield of the subtelomeric FISH method. A clinical checklist was developed to preselect patients for investigation. The selection criteria included prenatal/postnatal growth retardation, the presence of dysmorphism and/or congenital anomalies, and a family history of ID [37]. We found that frequency of subtelomeric rearrangements in patients with ID and MCA/DF was higher than that of those patients with ID alone (8.5\% versus $2.9 \%)$. Our finding supported the suggestion that preselection of patients using the abovementioned checklist, or a similar one, for subtelomeric FISH may increase the positive findings in patients with ID.

\subsection{Clinical Features of Patients with Subtelomeric Rearrange-} ments. In this study, we detected two well-recognized syndromes, namely, $4 \mathrm{p}$ deletion syndrome or Wolf-Hirschhorn syndrome and 1p36 deletion syndrome, in Patient 1 and Patient 2, respectively. Both syndromes have overlapping clinical features, including growth retardation, variable degree of ID/developmental delay, structural brain abnormalities, hypotonia, seizures, skeletal abnormalities, congenital heart defects, and hearing loss [38-40]. However, they are clinically recognized by distinct facial features, such as a Greek warrior helmet appearance of the nose, along with prominent glabella in the $4 \mathrm{p}$ deletion syndrome, and straight eyebrows, deeply set eyes, and midface retrusion in the 1p36 deletion syndrome. Clinical features of these syndromes may be variable depending on the extent of the deletions, in addition to the number and significance of the deleted genes. The deletions can be detected by using standard karyotype analysis in $50-60 \%$ of patients with $4 \mathrm{p}$ deletion syndrome and $25 \%$ of patients with $1 \mathrm{p} 36$ deletion syndrome, while FISH and chromosomal microarray (CMA) can detect chromosomal rearrangements in over $95 \%$ of the patients $[38,39]$. In this study, our patients with $4 \mathrm{p}$ deletion and 1p36 deletion syndromes had subtle facial features, most likely because they had cryptic subtelomeric deletions.

Approximately half of the subtelomeric rearrangements detected in patients with ID were unbalanced derivative chromosomes. The presence of derivative chromosome results in deletion (i.e., partial monosomy) along with duplication (i.e., partial trisomy) of distinct subtelomere regions. Clinical features that presented in our patients with derivative chromosomes were influenced by the coexistence of two genomic imbalances, from which phenotypic consequence resulting from one type of genomic imbalance confounds the phenotypic consequence resulting from the other genomic imbalance. In addition, variable expressivity, incomplete penetrance, and the degree of skewed X-inactivation when X chromosome involved in the derivative chromosomes could have an influence on the phenotypes of the patients.

Patient 3 possessed $\operatorname{der}(10) t(7 ; 10)(p 22.3 ; q 26.3)$, representing three $7 \mathrm{p}$ subtelomeres and only one $10 \mathrm{q}$ subtelomere. We found only one previous report of a patient with $\operatorname{der}(10) t(7 ; 10)(\mathrm{p} 22.3 ; \mathrm{q} 26.3)$. She was a 17 -year-old woman with short stature and moderate ID. Unlike our patient, she showed no dysmorphic facies and microcephaly [22]. Difference in clinical features may be due to the extent of the deletion and duplication. Duplication of 7p22.3 was reported on a patient with Asperger syndrome [41] and a patient with DF and skeletal abnormalities, including abnormal distal humeri [42]. A deformity of the elbow was present in our patient. In addition, developmental delay and minor DF were reported in the other patient with interstitial deletion of $10 \mathrm{q} 26.3$ [43].

Duplication of Xp22.3 that was detected in Patient 4 and Patient 5 was also detected in 2 out of 129 Thai patients with unexplained ID from a different cohort, who had subtelomeric rearrangements detected by MLPA technique [44]. Frequency of Xp22.3 duplication in Thai patients with unexplained ID was 1.9\% (4/211). The duplication of Xp22.3 was observed in individuals with neurocognitive and behavioral abnormalities [45]. Moreover, deletion of Xp22.3 was associated with $\mathrm{X}$-linked mental retardation and attention deficit hyperactivity disorder [45]. Patient 4 with deletion of 20 q13.3 had only moderate ID with minor DF. Deletion of 20 q13.33 was previously reported on patients with epileptic seizures. For Patient 5, significance of 3p26.3 deletion was unclear. Deletion of this region was previously described in patients with ID and atypical autism [46]; however, it was also reported in four generations of a family that were apparently healthy [47].

\section{Conclusions}

We detected subtelomeric rearrangements in $6.1 \%$ of the patients with ID. The sensitivity of subtelomeric FISH increases when preselection criteria were applied. We reported clinical entities observed in a patient with $\operatorname{der}(20) \mathrm{t}(\mathrm{X} ; 20)(\mathrm{p} 22.3 ; \mathrm{q} 13.3)$, a patient with $\operatorname{der}(3) \mathrm{t}(\mathrm{X} ; 3)(\mathrm{p} 22.3 ; \mathrm{p} 26.3)$, and a patient with $\operatorname{der}(10) t(7 ; 10)(p 22.3 ; q 26.3)$. These rearrangements have never been or had rarely been reported in literature. Even though 


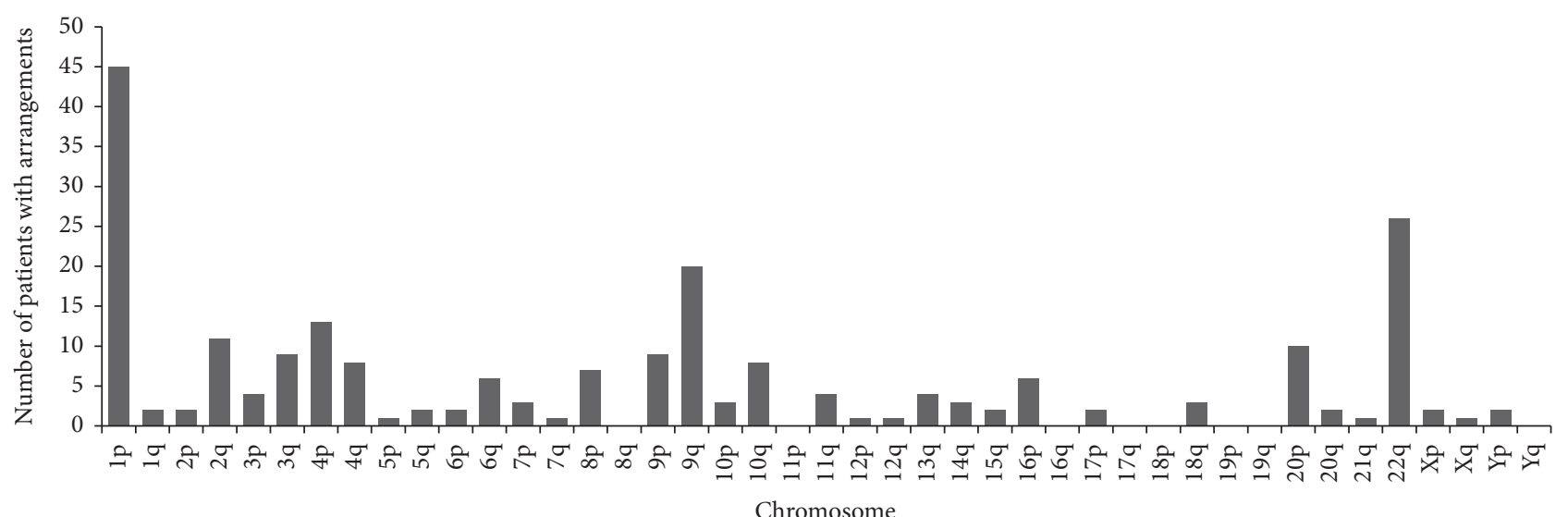

(a)

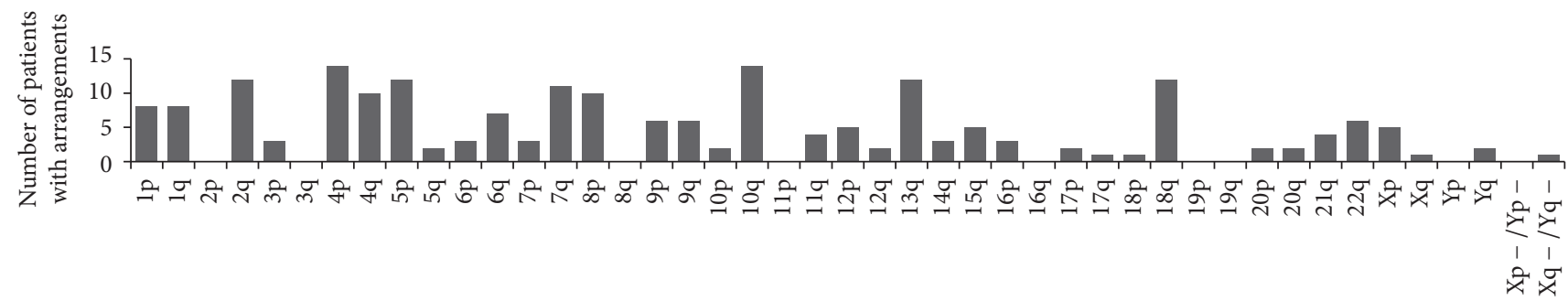

Chromosome

(b)

FigURE 3: Number of patients with subtelomeric rearrangements involving each chromosome region. (a) Terminal deletion $(n=226)$. (b) Unbalanced derivative. The only chromosome in which the subtelomere region was monosomy is depicted here $(n=204)$.

subtelomeric FISH is a very robust technique, as it can detect and locate rearrangements that involve small and specific regions of the chromosomes, uncover low level mosaicism, and identify balanced chromosomal rearrangements, CMA is currently recommended as a first-tier test and replaces the standard karyotype and subtelomeric FISH analyses for patients with ID of unknown causes [48]. However, in countries where CMA is unavailable or unaffordable, FISH is a useful technique to screen for subtelomeric rearrangements. In this study, we added information regarding clinical entities of our patients with subtelomeric rearrangements. This information requires accumulation from case reports and is useful for genetic counselling.

\section{Competing Interests}

All authors declare that they have no financial or other relevant conflict of interests regarding this paper.

\section{Acknowledgments}

The authors thank all participating families for their generous contribution of time and biological materials. They appreciate all the clinicians for their collaboration. This study is supported by the Thailand Research Fund (Grant no. PDF/98/2544), the National Center for Genetic Engineering and Biotechnology (Grant no. BT-B-01-MG-79-4701), and the Graduate School Research Dissertation Funding, Prince of Songkla University.

\section{References}

[1] WHO, ICD-10 Guide for Mental Retardation, World Health Organization, Geneva, Switzerland, 1996.

[2] J. Xu and Z. Chen, "Advances in molecular cytogenetics for the evaluation of mental retardation," American Journal of Medical Genetics Part C, vol. 117, no. 1, pp. 15-24, 2003.

[3] H. C. Mefford and B. J. Trask, "The complex structure and dynamic evolution of human subtelomeres," Nature Reviews Genetics, vol. 3, no. 2, pp. 91-102, 2002.

[4] S. J. L. Knight, R. Regan, A. Nicod et al., "Subtle chromosomal rearrangements in children with unexplained mental retardation," The Lancet, vol. 354, no. 9191, pp. 1676-1681, 1999.

[5] C. A. Joyce, N. R. Dennis, S. Cooper, and C. E. Browne, "Subtelomeric rearrangements: results from a study of selected and unselected probands with idiopathic mental retardation and control individuals by using high-resolution G-banding and FISH," Human Genetics, vol. 109, no. 4, pp. 440-451, 2001.

[6] Y.-S. Fan, Y. Zhang, M. Speevak, S. Farrell, J. H. Jung, and V. M. Siu, "Detection of submicroscopic aberrations in patients with unexplained mental retardation by fluorescence in situ hybridization using multiple subtelomeric probes," Genetics in Medicine, vol. 3, no. 6, pp. 416-421, 2001.

[7] C. Sismani, J. A. L. Armour, J. Flint, C. Girgalli, R. Regan, and P. C. Patsalis, "Screening for subtelomeric chromosome 
abnormalities in children with idiopathic mental retardation using multiprobe telomeric FISH and the new MAPH telomeric assay," European Journal of Human Genetics, vol. 9, no. 7, pp. 527-532, 2001.

[8] B.-M. Anderlid, J. Schoumans, G. Annerén et al., "Subtelomeric rearrangements detected in patients with idiopathic mental retardation," American Journal of Medical Genetics, vol. 107, no. 4, pp. 275-284, 2002.

[9] E. Baker, L. Hinton, D. F. Callen et al., "Study of 250 children with idiopathic mental retardation reveals nine cryptic and diverse subtelomeric chromosome anomalies," American Journal of Medical Genetics Part A, vol. 107, no. 4, pp. 285-293, 2002.

[10] S. Popp, B. Schulze, M. Granzow et al., "Study of 30 patients with unexplained developmental delay and dysmorphic features or congenital abnormalities using conventional cytogenetics and multiplex FISH telomere (M-TEL) integrity assay," Human Genetics, vol. 111, no. 1, pp. 31-39, 2002.

[11] A. J. Dawson, S. Putnam, J. Schultz et al., "Cryptic chromosome rearrangements detected by subtelomere assay in patients with mental retardation and dysmorphic features," Clinical Genetics, vol. 62, no. 6, pp. 488-494, 2002.

[12] B. Clarkson, K. Pavenski, L. Dupuis et al., "Detecting rearrangements in children using subtelomeric FISH and SKY," American Journal of Medical Genetics, vol. 107, no. 4, pp. 267-274, 2002.

[13] C. D. M. van Karnebeek, C. Koevoets, S. Sluijter et al., "Prospective screening for subtelomeric rearrangements in children with mental retardation of unknown aetiology: the Amsterdam experience," Journal of Medical Genetics, vol. 39, no. 8, pp. 546553, 2002.

[14] M. Kirchhoff, S. Pedersen, E. Kjeldsen et al., "Prospective study comparing HR-CGH and subtelomeric FISH for investigation of individuals with mental retardation and dysmorphic features and an update of a study using only HR-CGH," American Journal of Medical Genetics, vol. 127, no. 2, pp. 111-117, 2004.

[15] E. Font-Montgomery, D. D. Weaver, L. Walsh, C. Christensen, and V. C. Thurston, "Clinical and cytogenetic manifestations of subtelomeric aberrations: report of six cases," Birth Defects Research Part A-Clinical and Molecular Teratology, vol. 70, no. 6, pp. 408-415, 2004.

[16] S. Walter, C. Sandig, G. K. Hinkel et al., "Subtelomere FISH in 50 children with mental retardation and minor anomalies, identified by a checklist, detects 10 rearrangements including a de novo balanced translocation of chromosomes 17p13.3 and 20q13.33," American Journal of Medical Genetics, vol. 128, no. 4, pp. 364-373, 2004.

[17] L. Rodriguez-Revenga, C. Badenas, A. Sánchez et al., "Cryptic chromosomal rearrangement screening in 30 patients with mental retardation and dysmorphic features," Clinical Genetics, vol. 65, no. 1, pp. 17-23, 2004.

[18] E. Bocian, Z. Hélias-Rodzewicz, K. Suchenek et al., "Subtelomeric rearrangements: results from FISH studies in 84 families with idiopathic mental retardation," Medical Science Monitor, vol. 10, no. 4, pp. CR143-CR151, 2004.

[19] G. V. N. Velagaleti, S. S. Robinson, B. M. Rouse, V. S. Tonk, and L. H. Lockhart, "Subtelomeric rearrangements in idiopathic mental retardation," Indian Journal of Pediatrics, vol. 72, no. 8, pp. 679-685, 2005.

[20] A. Baroncini, F. Rivieri, A. Capucci et al., "FISH screening for subtelomeric rearrangements in 219 patients with idiopathic mental retardation and normal karyotype," European Journal of Medical Genetics, vol. 48, no. 4, pp. 388-396, 2005.
[21] M. Sogaard, Z. Tümer, H. Hjalgrim et al., "Subtelomeric study of 132 patients with mental retardation reveals 9 chromosomal anomalies and contributes to the delineation of submicroscopic deletions of 1pter, 2qter, 4pter, 5qter and 9qter," BMC Medical Genetics, vol. 6, article 21, 2005.

[22] S. Yu, E. Baker, L. Hinton et al., "Frequency of truly cryptic subtelomere abnormalities-a study of 534 patients and literature review," Clinical Genetics, vol. 68, no. 5, pp. 436-441, 2005.

[23] A. Erjavec-Škerget, Š. Stangler-Herodež, A. Zagorac, B. Zagradišnik, and N. Kokalj-Vokač, "Subtelomeric chromosome rearrangements in children with idiopathic mental retardation: applicability of three molecular-cytogenetic methods," Croatian Medical Journal, vol. 47, no. 6, pp. 841-850, 2006.

[24] M. Palomares, A. Delicado, P. Lapunzina et al., "MLPA vs multiprobe FISH: comparison of two methods for the screening of subtelomeric rearrangements in 50 patients with idiopathic mental retardation," Clinical Genetics, vol. 69, no. 3, pp. 228-233, 2006.

[25] A. Rauch, J. Hoyer, S. Guth et al., "Diagnostic yield of various genetic approaches in patients with unexplained developmental delay or mental retardation," American Journal of Medical Genetics, Part A, vol. 140, no. 19, pp. 2063-2074, 2006.

[26] J. B. Ravnan, J. H. Tepperberg, P. Papenhausen et al., "Subtelomere FISH analysis of 11688 cases: an evaluation of the frequency and pattern of subtelomere rearrangements in individuals with developmental disabilities," Journal of Medical Genetics, vol. 43, no. 6, pp. 478-489, 2006.

[27] G. E. Ütine, T. Çelik, Y. Alanay et al., "Subtelomeric rearrangements in mental retardation: Hacettepe University experience in 130 patients," The Turkish Journal of Pediatrics, vol. 51, no. 3, pp. 199-206, 2009.

[28] E. Mihçi, M. Özcan, S. Berker-Karaüzüm et al., "Subtelomeric rearrangements of dysmorphic children with idiopathic mental retardation reveal 8 different chromosomal anomalies," The Turkish Journal of Pediatrics, vol. 51, no. 5, pp. 453-459, 2009.

[29] E. F. Belligni, E. Biamino, C. Molinatto et al., "Subtelomeric FISH analysis in 76 patients with syndromic developmental delay/intellectual disability," Italian Journal of Pediatrics, vol. 35, article 9, 2009.

[30] J. Bogdanowicz, B. Pawłowska, A. Ilnicka et al., "Subtelomeric rearrangements in Polish subjects with intellectual disability and dysmorphic features," Journal of Applied Genetics, vol. 51, no. 2, pp. 215-217, 2010.

[31] S. R. dos Santos and D. V. Freire-Maia, "Absence of subtelomeric rearrangements in selected patients with mental retardation as assessed by multiprobe T FISH," Journal of Negative Results in BioMedicine, vol. 11, no. 1, article 16, 2012.

[32] J. W. Ahn, C. Mackie Ogilvie, A. Welch et al., "Detection of subtelomere imbalance using MLPA: validation, development of an analysis protocol, and application in a diagnostic centre," BMC Medical Genetics, vol. 8, article 9, 2007.

[33] A. P. A. Stegmann, L. M. H. Jonker, and J. J. M. Engelen, "Prospective screening of patients with unexplained mental retardation using subtelomeric MLPA strongly increases the detection rate of cryptic unbalanced chromosomal rearrangements," European Journal of Medical Genetics, vol. 51, no. 2, pp. 93-105, 2008.

[34] F. E. P. Mundhofir, W. M. Nillesen, B. W. M. Van Bon et al., "Subtelomeric chromosomal rearrangements in a large cohort of unexplained intellectually disabled individuals in Indonesia: a clinical and molecular study," Indian Journal of Human Genetics, vol. 19, no. 2, pp. 171-178, 2013. 
[35] B. C. Ballif, S. G. Sulpizio, R. M. Lloyd et al., "The clinical utility of enhanced subtelomeric coverage in array CGH," American Journal of Medical Genetics Part A, vol. 143, no. 16, pp. 18501857, 2007.

[36] A.-C. Thuresson, M.-L. Bondeson, C. Edeby et al., "Wholegenome array-CGH for detection of submicroscopic chromosomal imbalances in children with mental retardation," Cytogenetic and Genome Research, vol. 118, no. 1, pp. 1-7, 2007.

[37] B. B. A. De Vries, S. M. White, S. J. L. Knight et al., "Clinical studies on submicroscopic subtelomeric rearrangements: a checklist," Journal of Medical Genetics, vol. 38, no. 3, pp. 145150, 2001.

[38] A. Battaglia, J. C. Carey, and S. T. South, "Wolf-Hirschhorn syndrome," in GeneReviews ${ }^{\circledR}$, R. A. Pagon, M. P. Adam, H. H. Ardinger et al., Eds., University of Washington, Seattle, Seattle, Wash, USA, 1993-2016.

[39] A. Battaglia, “1p36 deletion syndrome," in GeneReviews ${ }^{\circledR}$, R. A. Pagon, M. P. Adam, H. H. Ardinger et al., Eds., University of Washington, Seattle, Seattle, Wash, USA, 1993-2016.

[40] V. K. Jordan, H. P. Zaveri, and D. A. Scott, "1p36 deletion syndrome: an update," The Application of Clinical Genetics, vol. 8, pp. 189-200, 2015.

[41] A. T. Vulto-van Silfhout, A. P. M. de Brouwer, N. de Leeuw, C. C. Obihara, H. G. Brunner, and B. B. A. de Vries, "A 380-kb duplication in 7p22.3 encompassing the LFNG gene in a boy with asperger syndrome," Molecular Syndromology, vol. 2, no. 6, pp. 245-250, 2012.

[42] K. M. Girisha, H. Abdollahpour, H. Shah et al., "A syndrome of facial dysmorphism, cubital pterygium, short distal phalanges, swan neck deformity of fingers, and scoliosis," American Journal of Medical Genetics Part A, vol. 164, no. 4, pp. 1035-1040, 2014.

[43] I. Y. Iourov, S. G. Vorsanova, O. S. Kurinnaia, and Y. B. Yurov, "An interstitial deletion at 10q26.2q26.3," Case Reports in Genetics, vol. 2014, Article ID 505832, 3 pages, 2014.

[44] S. Rujirabanjerd, O. Plong-On, T. Sripo, and P. Limprasert, "Subtelomeric aberrations in Thai patients with idiopathic mental retardation and autism detected by multiplex ligationdependent probe amplification," Asian Biomedicine, vol. 9, no. 4, pp. 501-509, 2015.

[45] P. Liu, A. Erez, S. C. S. Nagamani et al., "Copy number gain at Xp22.31 includes complex duplication rearrangements and recurrent triplications," Human Molecular Genetics, vol. 20, no. 10, pp. 1975-1988, 2011.

[46] A. A. Kashevarova, L. P. Nazarenko, S. Schultz-Pedersen et al., "Single gene microdeletions and microduplication of 3p26.3 in three unrelated families: CNTN6 as a new candidate gene for intellectual disability," Molecular Cytogenetics, vol. 7, article 97, 2014.

[47] S. Moghadasi, A. van Haeringen, L. Langendonck, A. C. J. Gijsbers, and C. A. L. Ruivenkamp, "A terminal 3p26.3 deletion is not associated with dysmorphic features and intellectual disability in a four-generation family," American Journal of Medical Genetics Part A, vol. 164, no. 11, pp. 2863-2868, 2014.

[48] J. B. Moeschler, M. Shevell, and Committee on Genetics, "Comprehensive evaluation of the child with intellectual disability or global developmental delays," Pediatrics, vol. 134, no. 3, pp. e903-e918, 2014. 

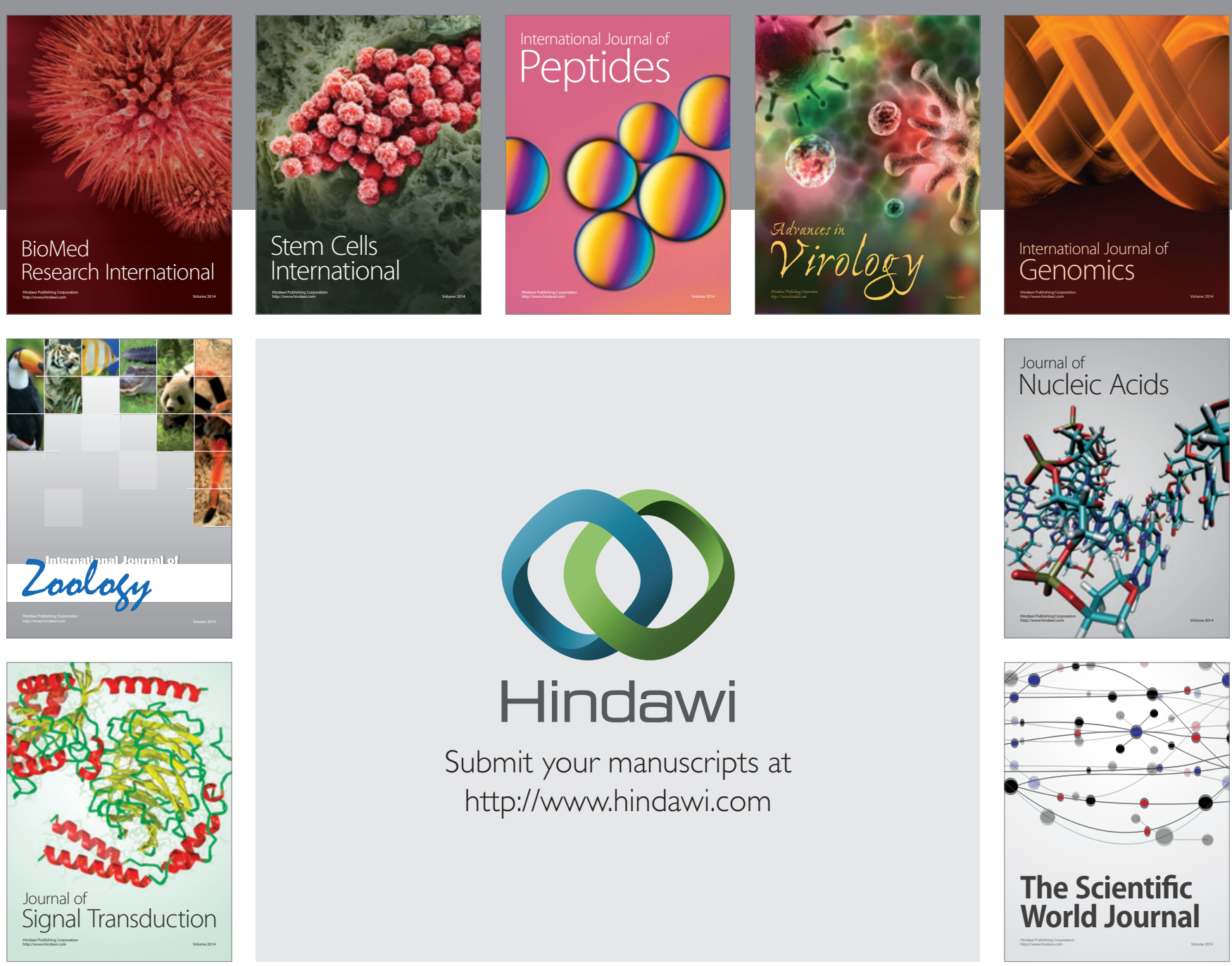

Submit your manuscripts at

http://www.hindawi.com
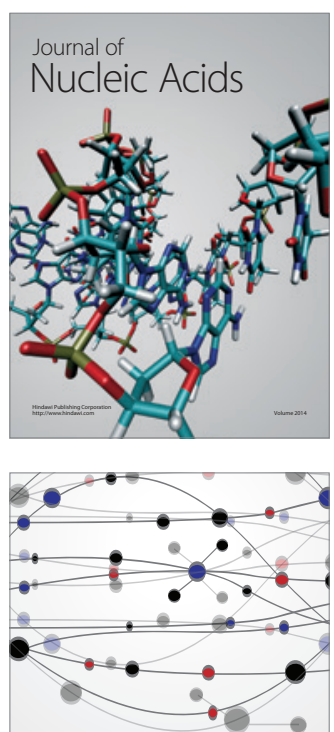

The Scientific World Journal
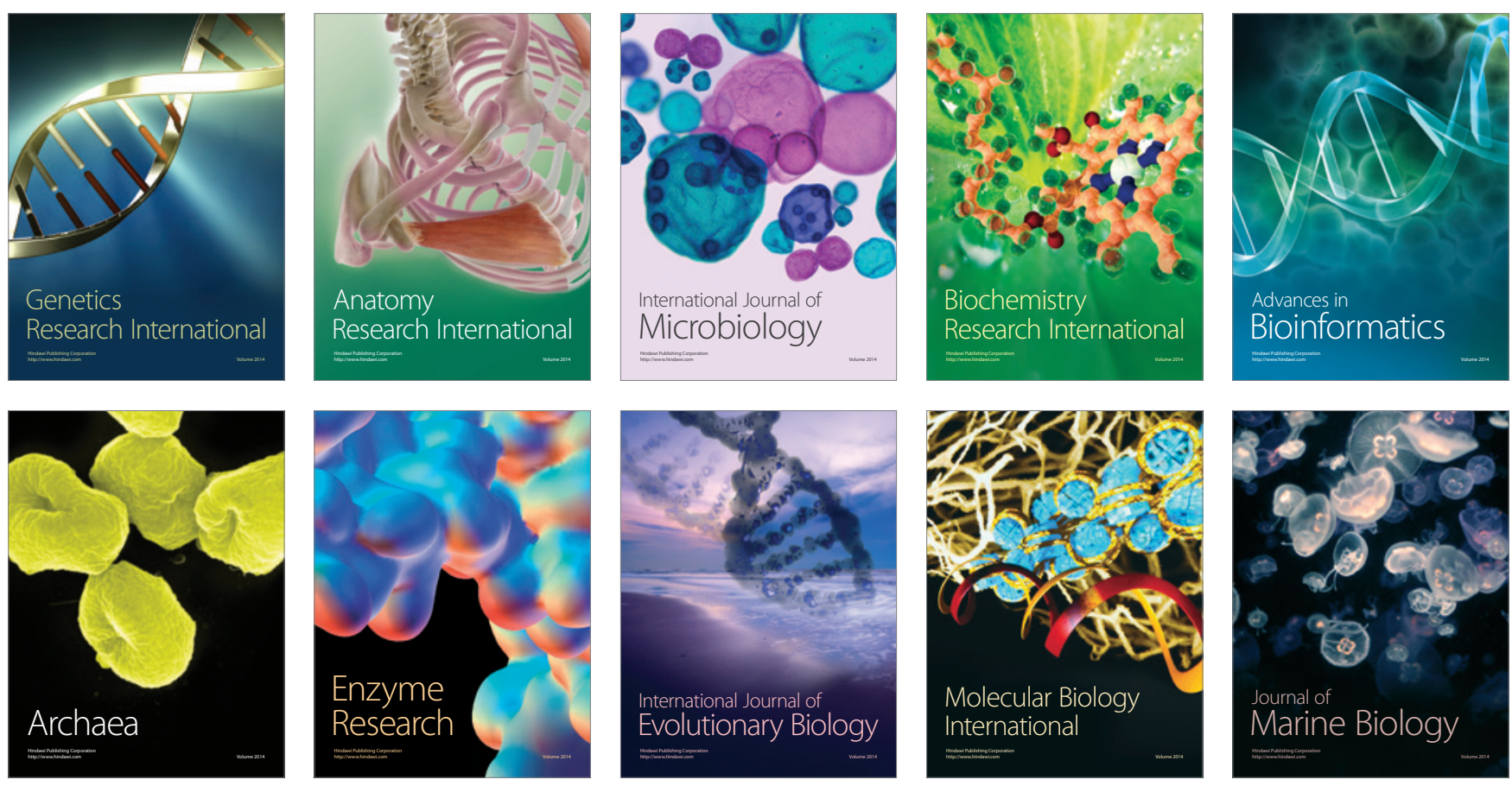